Classification

Physics Abstracts

$82.80 \mathrm{P}-68.55-61.80 \mathrm{~F}$

\title{
Croissance de films ultra-minces d'oxydes en surface du silicium (100) activée par bombardement électronique
}

\author{
Benasgoul Sefsaf, Bernard Carrière et Jean Paul Deville
}

I.P.C.M.S., Groupe Surfaces-Interfaces, U.M. CNRS - ULP - EHICS 46, 4 rue Blaise Pascal, F-67070 Strasbourg, France

(Reçu 2 janvier 1992, accepté le 1 avril 1992)

\begin{abstract}
Résumé. - Les films ultra-minces de silice sont obtenus à température ambiante et sous faisceau d'électrons primaires du spectromètre Auger. L'avantage de cette procédure est de pouvoir contrôler en continu la formation de l'interface $\mathrm{SiO}_{2}-\mathrm{Si}$. L'enregistrement étape par étape des spectres Auger AES (Auger Electron Spectroscopy) et de pertes d'énergies EELS (Electron Energy Loss Spectroscopy) nous permet d'analyser les modifications de liaisons chimiques dans l'interface formée, à partir des cinétiques d'oxydation et de l'évolution de la structure fine du spectre Auger $\mathrm{SiL}_{23} \mathrm{VV}$. Par ailleurs cette étude confirme qu'il est possible de faire croître à température ambiante des couches ultra-minces de $\mathrm{SiO}_{2}$, en surface du silicium, en activant l'oxydation par bombardement électronique.
\end{abstract}

\begin{abstract}
Ultra-thin layers of silica are obtained at room temperature by a mild oxidation process of silicon crystal surfaces. The growth is activated by the electron beam of an Auger spectrometer. It is thus possible to characterize step by step the building of $\mathrm{SiO}_{2}-\mathrm{Si}(100)$ interfaces. Oxidation kinetics and evolution of the Auger peak fine structure are used to study the reactivity of silicon to oxygen as a function of the surface crystalline order. The aim of the present paper is to describe the early stages of electron-beam induced oxide growth on $\mathrm{Si}(100)$ and to characterize the various silicon-oxygen chemical bonds at the $\mathrm{SiO}_{2}$-Si interface during the growth process.
\end{abstract}

\section{Introduction.}

L'oxydation de silicium a fait l'objet ces quinze dernières années d'un nombre considérable d'études fondamentales et appliquées, en raison de l'importance technologique qu'a pris ce matériau dans les domaines de la microélectronique et du photovoltaïque, importance liée aux propriétés diélectriques du dioxyde de silicium $\mathrm{SiO}_{2}$. Dans ce contexte, il est particulièrement intéressant de pouvoir réaliser des couches ultra-minces d'oxyde $(\leq 10 \mathrm{~nm})$. Or les procédés classiques d'oxydation thermique à haute température sont peu adaptés à la maîtrise de la croissance de couches d'une si faible épaisseur, sauf à travailler en oxygène dilué.

Une méthode alternative qui n'a pas encore fait ses preuves à l'échelle industrielle, pourrait être de réaliser l'oxydation à température ambiante, ce qui doit permettre également d'éviter la modification des caractéristiques électroniques intrinsèques du substrat silicium observée pour 
des oxydations pratiquées à plus haute température. Enfin, une telle méthode en jouant sur le balayage du faisceau autoriserait la gravure.

Des premières études sur l'oxydation de surfaces de Si(111) ont montré qu'il était possible d'obtenir des couches de quelques dizaines d' $\AA \AA$ d'épaisseur en maintenant le substrat de silicium sous bombardement électronique à faible pression d'oxygène $\left(\leq 10^{-6}-10^{-7}\right.$ torr $)[1,7,10]$.

Ceci peut être en particulier réalisé en utilisant le faisceau primaire d'électrons d'un analyseur Auger, ce qui permet corrélativement de suivre au moyen de cette spectroscopie le processus d'oxydation.

Dans cet article, nous présentons les résultats obtenus en utilisant la même approche expérimentale pour la surface (100) de silicium.

\section{Dispositif experimental.}

Les expériences sont faites dans une chambre d'analyse ultravide, dotée d'un spectromètre Auger de type CMA (Cylindrical Mirror Analyser).

Les échantillons (type N, 2 à $5 \Omega \mathrm{cm}$ ) sont des plaquettes de silicium monocristallin d'épaisseur de $100 \mu \mathrm{m}$. Le degré d'ordre cristallin et la propreté de la surface sont déterminés par LEED (Low Electron Energy Diffraction), AES (Auger Electron Spectroscopy) et EELS (Electron Energy Loss Spectroscopy).

La décontamination de la surface est obtenue par une succession de bombardements ioniques d'Argon et de recuits. La surface de $\mathrm{Si}(100)$ est ensuite maintenue sous le faisceau électronique propre de l'analyseur Auger (énergie primaire $E_{\mathrm{p}}=1,5 \mathrm{keV}$, courant primaire $l_{\mathrm{p}}=3 \mu \mathrm{A}$ $\left(5 \mathrm{~mA} \cdot \mathrm{cm}^{-2}\right)$. L'oxydation est réalisée à température ambiante sous une pression partielle d'oxygène de $2 \times 10^{-7}$ torr. Les spectres LVV et KLL du silicium et de l'oxygène enregistrés en continu sont caractéristiques du processus d'oxydation superficielle observée sous bombardement d'électrons.

\section{Résultats.}

3.1 Cinétiques de CROISSANCE AST (Auger Signal vs. Time). - Selon l'approche mise en oeuvre pour la face (111) [1], l'oxydation de la surface reconstruite $\mathrm{Si}(100)-(2 \times 1)$ [2] peut être décrite en considérant l'évolution des intensités des pics Auger du substrat ( $\mathrm{SiL}_{23} \mathrm{VV}$ à $92 \mathrm{eV}$ ) et de l'adsorbat oxygène (OKLL à $505 \mathrm{eV}$ ) en fonction de la durée d'irradiation sous pression d'oxygène $\left(2 \times 10^{-7}\right.$ torr $)$.

Nous présentons sur la figure 1 les spectres Auger $E \cdot \mathrm{d} N / \mathrm{d} E$ du silicium relatifs au silicium propre et à l'oxyde formé au bout de 3 heures, ces deux situations étant empruntées à une des expériences.

Nous établissons les cinétiques comme suit : $I(\mathrm{Si})$, en ordonnées, désigne le rapport $h(\mathrm{Si}) / h_{0}(\mathrm{Si}), h_{0}(\mathrm{Si})$ étant l'intensité initiale du pic de silicium à $92 \mathrm{eV}, h(\mathrm{Si})$ son intensité à un instant $t$ donné quelconque. Nous portons en abscisse le temps d'oxydation.

La figure 2 présente deux cinétiques $I(\mathrm{Si})$ obtenues respectivement pour une oxydation sous bombardement électronique de la surface $\mathrm{Si}(100)-(2 \times 1)$ et de $\mathrm{Si}(111)-(7 \times 7)$. Ces deux surfaces sont décontaminées au préalable par abrasion ionique (argon) et recuites à $950^{\circ} \mathrm{C}$ sous ultravide. La cinétique $I(\mathrm{Si})$, dans le cas de la face (100), peut être décrite par quatre régions correspondant à trois changements de pente consécutifs, comme dans le cas de l'oxydation de la face monocristalline (111) [1]. 


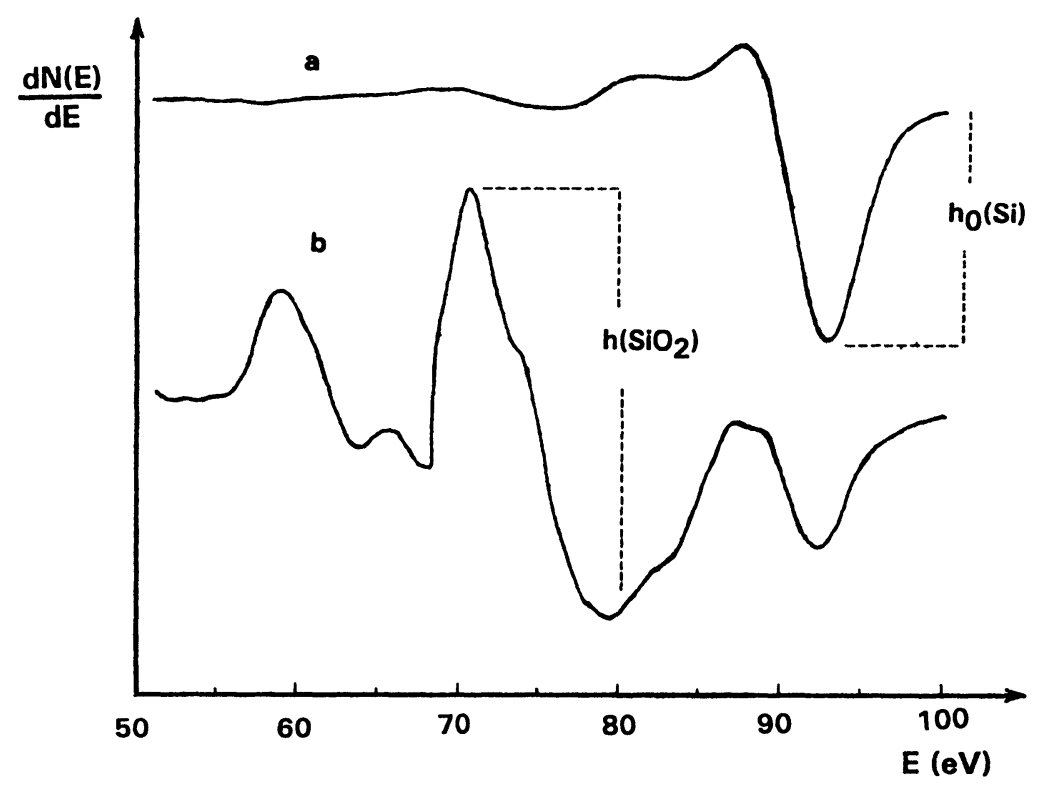

Fig. 1. - Spectres Auger SiLVV : a) surface propre, b) au terme de l'oxydation.

[SiLVV Auger spectra: a) clean surface, b) at the end of the oxidation.]

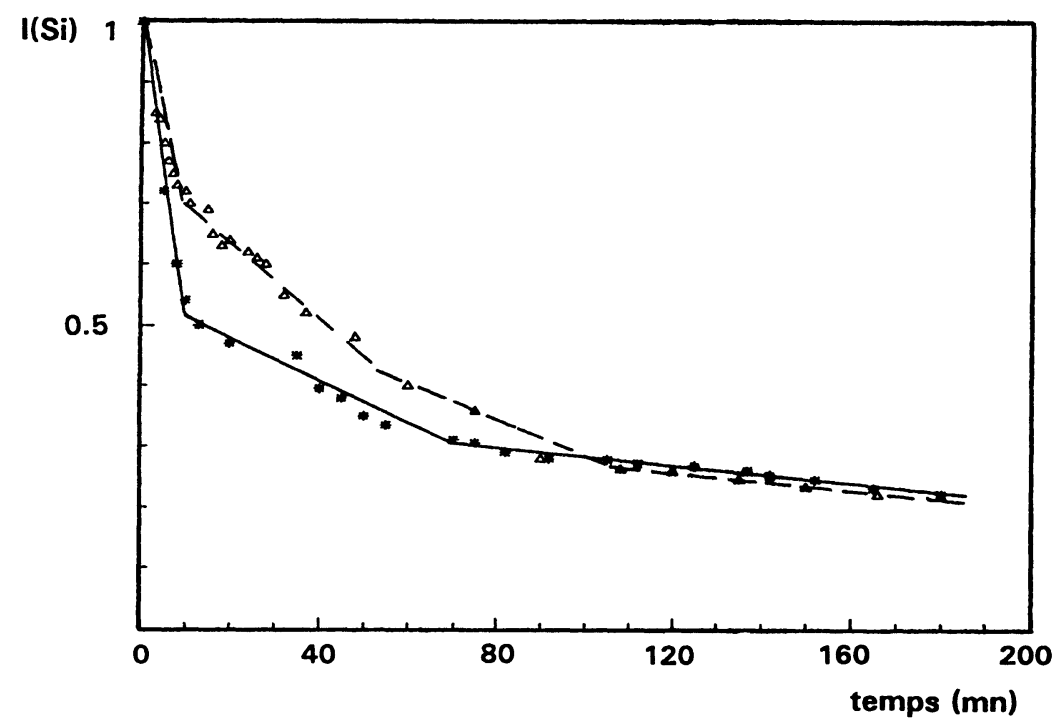

Fig. 2. - Cinétique d'oxydation sous faisceau électronique : $(\triangle)$ surface $\operatorname{Si}(100),(*)$ surface $\operatorname{Si}(111)$. [Kinetics of the electron beam induced oxidation : $(\Delta) \mathrm{Si}(100)$ surface, $(*) \mathrm{Si}(111)$ surface.]

La première brisure sur la cinétique de $\mathrm{Si}(100)$ est observée pour $I=0,7$ soit pour une épaisseur concernée par l'oxydation de l'ordre de $2 \AA$. Le deuxième changement de pente a lieu pour $I=0,48$, correspondant à une épaisseur équivalente d'oxyde de $3,7 \AA$. Une troisième 
brisure est observée pour $I(\mathrm{Si})=0,2$ soit pour une épaisseur équivalente de $8 \AA$. Au stade final atteint dans cette expérience, l'épaisseur équivalente est estimée à 11,5 $\AA$.

Les épaisseurs d'oxyde sont évaluées en appliquant la loi d'atténuation [3] $I(\mathrm{Si})=$ $\exp (-d / \lambda \cos \Theta)$, avec $d$ l'épaisseur équivalente d'oxyde et $\lambda$ le libre parcours moyen des électrons de $92 \mathrm{eV}$, que nous prendrons de l'ordre de $6,5 \AA[4,5]$. L'application de cette loi d'atténuation n'est justifiée que dans le cas d'une croissance homogène, par exemple du type couche par couche $[6,7]$. En conséquence, le calcul des épaisseurs concernées par l'oxydation n'est fournie que comme un critère d'appréciation de l'importance de l'oxydation.

En fait, la nature du recouvrement en oxyde est très liée à l'état structural de la surface initiale du silicium [8]. Ainsi pour une surface désordonnée par bombardement ionique, le recouvrement a tendance à être moins homogène que sur une surface reconstruite $[1,9]$ et cela a été confirmé dans le cadre de cette étude, pour l'oxydation de la face (100).

Nous avons également porté sur la même figure les résultats d'une étude comparative réalisée dans les mêmes conditions expérimentales sur la face (111). Les différences de comportement des deux faces (100) et (111) sont à chercher dans les atténuations mises en jeu et la cinétique d'apparition des changements de pente. Les premiers changements de pente (développement de la structure $\alpha$ qui traduit le début de la croissance des tétraèdres $\mathrm{SiO}_{4}$ ) se produisent pratiquement pour la même durée d'oxydation. Cependant, les valeurs de $I(\mathrm{Si})$ ne sont pas identiques: $I(\mathrm{Si})=$ 0,7 pour la face $(100), I(\mathrm{Si})=0,55$ pour la face $(111)$. Les épaisseurs équivalentes correspondantes sont de l'ordre de $2 \AA$ pour (100) et de 3,5 $\AA$ pour (111).

En fait, cette première observation traduit une différence de réactivité importante entre les deux faces, liée à la différence d'arrangement structural en surface. Si on admet que pour la face dense (111), l'atténuation du pic initial de Si correspond à un recouvrement homogène d'oxygène, on doit considérer que l'adsorption d'oxygène sur la face (100) se fait selon des sites préférentiels et que la croissance des tétraèdres $\mathrm{SiO}_{4}$ s'opère avant que le recouvrement par l'oxygène de cette face ne soit complet. Cette interprétation de la différence de réactivité initiale est cohérente avec le comportement ultérieur des deux surfaces.

La deuxième brisure (apparition de la structure $\beta$ accompagnant la croissance de silice tridimensionnelle) se produit beaucoup plus tôt sur la face (100). Si, au delà, la saturation du processus est plus marquée sur la face (111), au stade final de l'oxydation, les atténuations de l'intensité du pic du substrat sont tout à fait comparables dans les deux cas, et correspondent à des épaisseurs équivalentes de $10 \AA$ environ.

Ceci permet de mettre en évidence que si la réactivité à l'oxygène est très différente pour les deux faces aux premiers stades, tout se passe, pour une oxydation suffisamment poussée, comme si le matériau oubliait cette différence initiale.

3.2 Evolution de L'ÉnERgIE ET DE LA FORME DES RAIES AUgER. - La figure 3 montre l'évolution du spectre $\mathrm{SiL}_{23} \mathrm{VV}$ et de sa structure fine lors de l'oxydation de la face (100). Ces modifications dans la structure fine peuvent être corrélées aux trois changements de pente observés sur la figure 2, en respectant la classification adoptée pour $\mathrm{Si}(111)$ [1] :

1) La premiere observation est l'apparition dans toutes les premières phases de l'oxydation d'une structure à $84 \mathrm{eV}$. Cette structure à $84 \mathrm{eV}$ est attribuée à la formation d'un sous-oxyde $\mathrm{SiO}_{x}$ [10 - 12] de stoechiométrie moyenne proche de SiO.

Une analyse plus complète de cette structure à $84 \mathrm{eV}$ a montré qu'elle pourrait être attribuée à un état d'oxydation préalable à la croissance de la silice. Les résultats de photoémission [13, 14] pour l'oxydation de $\mathrm{Si}(100)$ permettent d'interpréter la structure à $84 \mathrm{eV}$ comme étant une transition croisée entre le substrat silicium et les atomes d'oxygène chimisorbés [1], mettant en jeu une lacune $2 p$ du substrat et 2 électrons non liants $02 p$.

Une autre interprétation [15] revient à faire intervenir à partir d'une lacune $2 p$ du silicium 


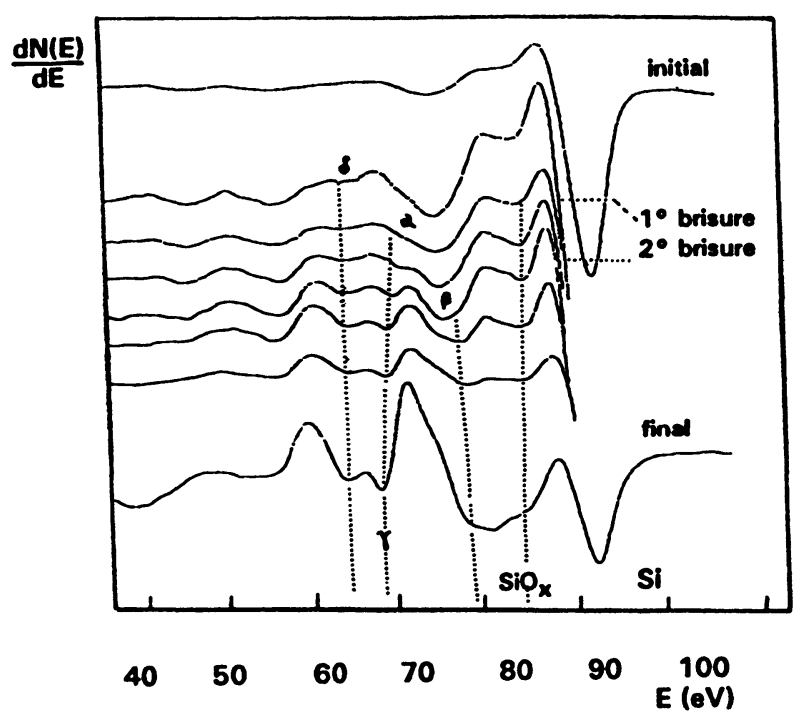

Fig. 3. - Evolution de la structure fine du spectre SiLVV au cours de l'oxydation. [Evolution of the SiLVV Auger fine structure during the oxidation.]

oxydé, un électron non liant $\mathrm{O} 2$ p et un électron liant. Dans cette première étape de l'oxydation, apparaît également une structure à $66 \mathrm{eV}$, notée $\delta$ qui va s'amplifier durant toute l'oxydation.

2) La deuxième étape de l'oxydation commence avec l'apparition de la structure appelée $\alpha$ à $71 \mathrm{eV}$; elle correspond au premier changement de pente. Par analogie avec l'étude de l'oxydation de la face $\mathrm{Si}(111)$, nous attribuons cette structure au début de la formation des tétraèdres $\mathrm{SiO}_{4}$ $[11,13]$. L'oxyde formé dans cette phase n'est pas stable à température ambiante sous ultra-vide ; nous le qualifions de silice perturbée ou de quasi-silice [6].

3) La troisième étape commence avec le deuxième changement de pente de la cinétique AST (Auger Signal vs. Time), soit au bout de deux heures environ d'oxydation. A ce stade, la structure $\beta$, qui apparait très nettement à 78,9 eV traduit la formation de la silice tridimensionnelle. Quand l'état final est atteint, dans les conditions de cette expérience, soit après trois heures d'oxydation, toutes les structures caractéristiques de la silice sont présentes dans le spectre Auger : $\beta$ à $78,5 \mathrm{eV}$, $\delta$ à $67,8 \mathrm{eV}, \gamma$ à $63,8 \mathrm{eV}$.

3.3 ROLE DU BOMBARDEMENTÉLECTRONIQUE. - L'influence du bombardement électronique dans le processus d'oxydation superficielle du silicium monocristallin, à température ambiante, a été établie expérimentalement dans des travaux antérieurs $[1,16,17]$, mais pour des conditions différentes d'une expérience à une autre. La visualisation du rôle du bombardement électronique peut être faite en établissant au terme de l'oxydation une topographie de la surface, latéralement de part et d'autre de la zone irradiée. Ceci est réalisé en enregistrant les spectres Auger des principaux pics $\left(\mathrm{Si}, \mathrm{SiO}_{2}, \mathrm{OKLL}\right)$ en différents points de la surface [18].

La figure 4a montre la topographie obtenue en portant les intensités du pic principal du substrat Si et de l'oxygène, en fonction de la position de la plage analysée. Sur la figure $4 \mathrm{~b}$, nous avons rassemblé les spectres $\mathrm{SiL}_{23} \mathrm{VV}$ relatifs aux trois régions principales de la topographie. On distingue trois régions, la première (I) correspondant à une couche d'oxyde d'épaisseur constante, la deuxième (II) rendant compte d'une diminution progressive d'efficacité des électrons, la troisième (III) pouvant être considérée comme exempte d'effets du bombardement électronique. On doit 
noter par ailleurs que le diamètre de la zone centrale oxydée est grand devant le diamètre du faisceau incident estimé à $0,05 \mathrm{~mm}$.

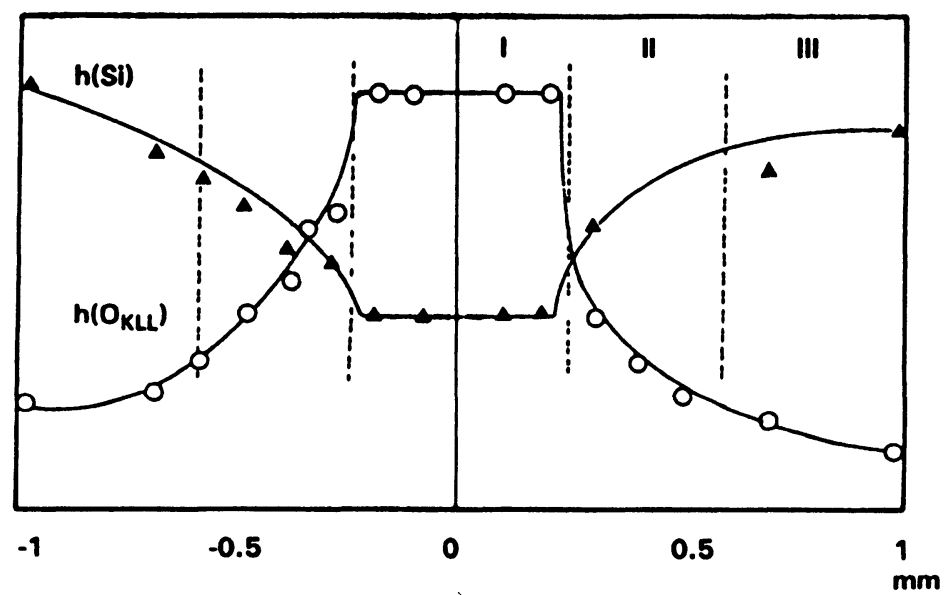

a)

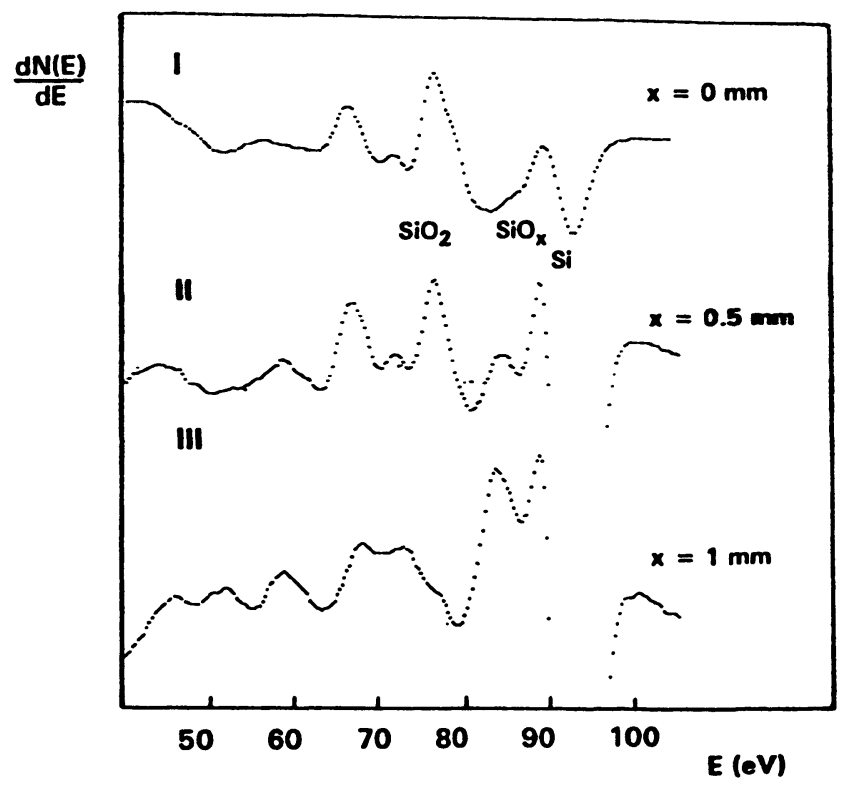

b)

Fig. 4. - a) Topographie de la surface oxydée sous faisceau électronique. b) Spectres Auger pour diverses plages analysees : I) plage irradiee, II) plage intermédiaire, III) plage non irradiée.

[a) Topography of the surface oxidized under electron beam. b) Auger spectra for various analyzed areas: I) area under irradiation, II) intermediate area, III) non irradiated area.]

Une telle observation ne contredit pas les hypothèses avancées dans les études de la réduction de la silice sous bombardement électronique $[6,20]$ et pour l'oxydation du silicium par une source 
de type plasmatron [20].

Dans les deux types d'études, il a été montré que des considérations de section efficace d'interaction et de dissociation permettent d'attribuer aux électrons secondaires (énergie $\leq 50 \mathrm{eV}$ ) le rôle déterminant dans la stimulation de l'oxydation superficielle sous faisceau d'électrons. Cette hypothèse est expérimentalement confirmée par l'existence d'une zone de transition de part et d'autre de la zone centrale d'impact d'une largeur compatible avec celle du cône de rétrodiffusion des électrons secondaires.

La comparaison de ces différents spectres confirme l'efficacité du bombardement électronique. Sur la plage non irradiée (III) sont observés les pics $\mathrm{SiO}_{x}, \alpha$ et $\delta$. C'est donć un stade qui précède la formation de la silice tridimensionnelle, analogue à une oxydation superficielle à température ambiante du silicium monocristallin, en l'absence du bombardement électronique. Par ailleurs, la topographie rend compte d'une symétrie dans le processus d'oxydation de part et d'autre de la plage centrale d'impact électronique [18].

En nous référant au modèle de Cabrera et Mott [21], deux mécanismes fondamentaux régissant la croissance d'un oxyde de silicium sous bombardement électronique peuvent être envisagés :

* une réaction de surface, entre les électrons incidents et l'oxygène, du type attachement dissociatif, et conduisant à la réaction d'une espèce oxydante réactive à basse température $: \mathrm{O}_{2}+\mathrm{e}^{-} \rightarrow$ $\mathrm{O}^{-}+\mathrm{O} \uparrow$

* un mécanisme de transport, sous champ électrique constant, de l'espèce oxydante créé en surface à partir de la réaction d'attachement dissociatif.

D'après Collot et al. [22], un mouvement de type "proche en proche" d'oxygène chargé est cohérent avec leurs expériences de traçage isotopique. On peut supposer que le bombardement électronique contribue à créer de nouveaux sites d'adsorption ou défauts, permettant d'accroître la fixation de l'oxygène dans la silice. Un tel mécanisme permet également d'expliquer la saturation du processus d'oxydation observé. Cette saturation pourrait être liée à la probabilité qu'ont les espèces oxydantes de joindre l'interface $\mathrm{SiO}_{2}-\mathrm{Si}$.

Nous appuyant sur Mott et Cabrera [21], un autre paramètre doit être invoqué, la diminution du champ électrique avec l'épaisseur de l'oxyde. Dès lors, on conçoit que l'épaisseur d'oxyde formé dépendra fortement de la pression d'oxygène, de la dose d'électrons incidents (en $\mathrm{mA} / \mathrm{cm}^{2}$ ) et de la densité d'électrons secondaires d'efficacité maximale (soit d'énergie voisine de $10 \mathrm{eV}$ ).

Collot et al. [22] ont prouvé directement l'efficacité des électrons de plus basse énergie (de l'ordre de $10 \mathrm{eV}$ ) et, pour les mêmes expositions à l'oxygène, l'efficacité d'une oxydation sous électrons (dose électronique : $1 \mathrm{~mA} / \mathrm{cm}^{2}$ ), à température ambiante, par rapport à des oxydations thermiques réalisées entre 700 et $900^{\circ} \mathrm{C}$.

\section{Conclusion.}

L'oxydation à température ambiante du silicium monocristallin ne permet pas d'aller au delà de la formation d'un oxyde dit "natif" dont l'épaisseur n'excède pas 8 à $10 \AA$. Pour obtenir à des températures ambiantes des épaisseurs d'oxyde compatibles avec leur utilisation dans des structures du type métal-oxyde-semiconducteur, une méthode revient à maintenir l'échantillon sous le bombardement électronique propre de l'analyseur Auger. Outre la possibilité d'obtenir, dans des conditions douces d'oxydation des épaisseurs d'oxydes (pression d'oxygène $\geq 10^{-7}$ torr, température ambiante) supérieures à $30 \AA$, cette approche permet, par l'exploitation qualitative et semi quantitative des spectres Auger enregistrés continûment, d'obtenir une description très détaillée de la formation de l'interface $\mathrm{SiO}_{2}-\mathrm{Si}(100)$.

A cette fin, un effort significatif a été consacré à l'exploitation des spectres Auger. L'analyse des modifications de la structure fine des transitions LVV du silicium a permis de comparer les 
principales étapes observées ici, avec celles obtenues lors de l'oxydation de la face (111):

1) Croissance d'un oxyde intermédiaire $\mathrm{SiO}_{x}$ (avec $x$ voisin de 1). 2) Formation de tétraèdres $\mathrm{SiO}_{4}$ et croissance d'une silice "perturbée" ou quasi silice, jusqu'à 10 Å d'épaisseur d'oxyde. 3) Croissance de la silice massive, $\mathrm{SiO}_{2}$.

L'étude des cinétiques d'oxydation et des formes des raies Auger pour les faces (100) et (111) a permis de faire plusieurs observations. L'examen des deux cinétiques laisse apparaître que dans ses premières phases l'oxydation semble se développer de façon plus homogène sur la face (111). La croissance ultérieure sur la face (100) présenterait un comportement comparable à celui d'une face (111) désordonnée.

Un prolongement de cette étude pourrait être l'obtention sous balayage électronique de couches ultra-minces d'oxydes de plus grande superficie. Il conviendra par ailleurs d'en tester les propriétés diélectriques au regard des performances recherchées. Nous n'oublions pas de mentionner une application potentielle et spécifique de ce procédé d'oxydation, en l'occurrence la réalisation de masques de $\mathrm{SiO}_{2}$ pour de futures nanostructures.

\section{Remerciements.}

Les auteurs remercient B. Lang pour l'aide qu'il leur a apportée dans l'analyse des résultats expérimentaux.

\section{Bibliographie}

[1] El MaACHI A., Thèse de Doctorat, Universite Strasbourg (1985).

[2] TROMP R.M., SMEENK R.G., SARIS F.W., ChADI D.J., Surf. Sci. 133 (1983) 137.

[3] MeYer F., VRaKKIng J.J., Surf. Sci. 38 (1973) 275.

[4] BRUNDLE C.R., Surf. Sci. 48 (1975) 99.

[5] DERRIEN J., COMMANDRE M., Surf. Sci. 118 (1982) 32.

[6 CARRIÈRE B., Thèse de Doctorat d'Etat, Strasbourg (1977).

[7] CARRIÈRE B., DEVILlE J.P., Surf. Sci. 80 (1979) 278.

[8] LANG B., SCHOller B., CARRIËRE B., Surf. Sci. 99 (1980) 103.

[9] Carrière B., Deville J.P., Sefsaf B., J. Phys. C (1986) 233.

[10] CARRIËRE B. et al, Microsc. Spectr. Electr. 3 (1978) 225.

[11] CARrière B. et al., Philos. Mag. B55 (1987) 721.

[12] KeIM E.G., VAN SILFHOUT A., Surf. Sci. 152/153 (1985) 1096.

[13] HOLLINGER G., Communication aux journées G. CIS Paris (1985).

[14] Hollinger G., Appl. Surf. Sci. 38 (1981) 318.

[15] JULIET P., Thèse de Doctorat Ingénieur, Grenoble (1984).

[16] CARrière B. et al., Surf. Sci. 126 (1983) 495.

[17] ChOUIYAKH A., Thèse de Doctorat d'Etat, Strasbourg (1984).

[18] SEFSAF B., Thèse de Doctorat, Université, Strasbourg (1989).

[19] CARRIÈre B., LANG B., Surf. Sci. 64, (1977) 209.

[20] Collơ P., Thèse de 3eme cycle, Paris (1985).

[21] CABrera N., MotT N.F., Rep. Prog. Phys. 12 (1949) 163.

[22] Collor P. et al, Philos. Mag. B55 (1987) 1059. 